\title{
Etudes contrastives des systèmes vocaliques français et vietnamien. Propositions méthodologiques pour l'apprenant vietnamien du FLE
}

\author{
Ly Nguyen \\ Ecole des Langues et des Etudes Internationales - Universite Nationale de Hanoi \\ nguyen.ngocluuly@yahoo.fr
}

La langue est un système de signalisation particulier, le moyen de communication le plus principal et le plus important des membres d'une communauté. En parlant du langage naturel, on doit bien distinguer le langage (aptitude particulière de l'homme de pouvoir exprimer leurs sentiments, leurs désirs ou leurs ordres au moyen d'une langue) avec les autres langues concrètes de chaque pays. Dans le monde, il s'agit de 3000 langues différentes, de différentes particularités, on ne peut pas identifier les façons d'enseignement de chaque langue. L'enseignement de l'anglais est tout à fait distinctif de celui du français, et n'est pas identique au japonais... Les professeurs de langues doivent donc fournir des remarques de différentes méthodes.

Avec l'enseignement des compétences linguistiques, celui des compétences communicatives comme la phonétique, le vocabulaire et la grammaire joue un rôle bien important. Dans les méthodes traditionnelles d'enseignement des langues, on met en exergue le contenu de la langue, des éléments segmentaux comme: phonèmes, morphèmes, mots, phrases... puis vocabulaire et grammaire et on fait peu de cas pour la forme de la langue. En fait, la phonétique est indispensable dans la communication, elle facilite surtout l'expression orale et la compréhension orale.

\section{Systèmes vocaliques français et vietnamien}

\subsection{Système vocalique français}

Il y a seize voyelles en français. Du point de vue articulatoire, les voyelles françaises se distinguent par:

- la résonance: on oppose les voyelles orales aux voyelles nasales. En français, on a les voyelles orales suivantes:

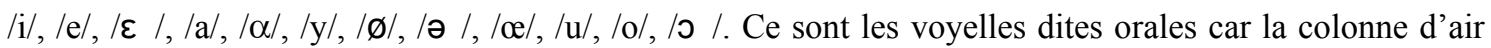
emprunte uniquement la cavité buccale (la luette s'élève pendant l'articulation).

Il y a encore quatre voyelles nasales: /ã/, /õ/, / $\mathfrak{e}^{\sim} /, / \varepsilon \%$. En les prononçant, la colonne d'air emprunte à la fois les cavités buccale et nasale (la luette s'abaisse pendant l'articulation de la voyelle, elle permet l'échappement d'une partie d'air venant des poumons par le nez).

- l'aperture: Selon le degré d'ouverture de la bouche, on a les voyelles d'aperture différente:

+les voyelles fermées: /i/, /y/, /u/

+les voyelles semi-fermées: /e/, /ø/,/o/, /0̃/

+la voyelle moyenne: /ə /

+les voyelles semi-ouvertes: $/ \varepsilon /, / \infty /, / 0 /, / \varepsilon \sim, / \propto^{\sim} /$ 
+les voyelles ouvertes: /a/, / $\alpha /, / \tilde{a} /$

- le lieu d'articulation: Selon que la masse de la langue s'avance ou recule dans la cavité buccale, on distingue les voyelles antérieures /i/, /e/, / $\varepsilon /, / \varepsilon /, / y /, / \varnothing /, / ə /, / œ /, / e^{\sim} /$ e t les voyelles postérieures /u/, $/ \mathrm{o} /, / \mathrm{o} /, / \mathrm{o} \% /, / \tilde{a} /$

- la labialisation: On oppose enfin, les voyelles dont l'articulation se fait avec ou sans l'arrondissement des lèvres. Ainsi, on distingue les voyelles écartées des voyelles arrondies. On a les voyelles écartées /i/, /e/, /e/; les voyelles arrondies /y/, /ø/, /œ/, /œ /, /u/, /o /, /o /, /0 \%

Observons le tableau ci-dessous:

\begin{tabular}{|c|c|c|c|c|}
\hline & $\begin{array}{l}\text { antérieures } \\
\text { écartées }\end{array}$ & $\begin{array}{l}\text { antérieures } \\
\text { arrondies }\end{array}$ & $\begin{array}{l}\text { postérieures } \\
\text { écartées }\end{array}$ & $\begin{array}{l}\text { postérieures } \\
\text { arrondies }\end{array}$ \\
\hline fermées & $\mathrm{i}$ & $\mathrm{y}$ & & $\mathrm{u}$ \\
\hline semi-fermées & $\mathrm{e}$ & $\varnothing$ & & $0 \quad$ \\
\hline moyenne & & $\partial$ & & \\
\hline \multirow[t]{2}{*}{ Semi-ouvertes } & $\varepsilon$ & $œ$ & & 0 \\
\hline & $\varepsilon^{\sim}$ & $œ^{\sim}$ & & \\
\hline ouvertes & $\mathrm{a}$ & & $\mathrm{a} \tilde{a}$ & \\
\hline
\end{tabular}

\subsection{Système vocalique vietnamien}

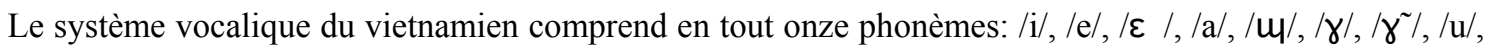
/o/, /o /, / ̃̃/.

Les différentes particularités articulatoires de ces voyelles peuvent se résumer dans le tableau suivant:

\begin{tabular}{|l|l|l|l|}
\hline & $\begin{array}{l}\text { antérieures } \\
\text { non-arrondies }\end{array}$ & $\begin{array}{l}\text { moyennes } \\
\text { non-arrondies }\end{array}$ & $\begin{array}{l}\text { postérieures } \\
\text { arrondies }\end{array}$ \\
\hline fermées & $\mathrm{i}$ & $\mathrm{u}$ & $\mathrm{u}$ \\
\hline semi-fermées & $\mathrm{e}$ & $\mathrm{0}$ \\
\hline semi-ouvertes & E & \\
\hline
\end{tabular}




\begin{tabular}{|l|l|}
\hline ouvertes & a \\
\end{tabular}

Les phonèmes $/ \mathrm{i} /, / \mathrm{e} /, / \varepsilon /, / \mathrm{a} /, / \mathrm{u} /, / \mathrm{o} /, / \mathrm{O} /$ se réalisent à peu près comme leurs homologues respectifs français.

Les quatre phonèmes $/ u /, / \gamma /, / \gamma /, / a /$ n'existent pas dans le système phonémique français:

Le phonème / $\mathrm{u} /$ se réalise comme une voyelle non-arrondie d'aperture minimum.

Le phonème $/ \gamma /$ est un peu plus postérieur et plus fermé que le phonème /ə / en français. Il se réalise comme une voyelle moyenne non-arrondie d'aperture du deuxième degré.

Les phonèmes $/ \gamma^{\sim} /$ et $/ a /$ sont respectivement les formes brèves des phonèmes $/ \gamma /$ et $/ a /$ le signe diacritique marque le trait bref d'un phonème). Ces deux phonèmes brefs ne se trouvent jamais seuls. Ils ne peuvent se réaliser que grâce à la combinaison avec d'autres phonèmes pour former un groupe.

On ne trouve jamais les mots "â", "ă" seuls dans les phrases mais "ớ", "á". Sauf $/ \gamma$ / $/$ ă/, chaque phonème- voyelle en vietnamien peut former à lui seul un élément signifiant. On y voit nettement la coïncidence, souvent constatée dans cette langue, des unités de dimensions différentes: le phonème, le morphème et le mot.

$$
\begin{aligned}
& \text { Exemple: } \mathrm{y} / \mathrm{i}^{1} / \text { médecine } \\
& \text { ô } / 0^{4} / \text { nid } \\
& o / \gamma^{6} / \text { éructer } \\
& \text { ề } / \mathrm{e}^{5} / \text { immariable } \\
& \text { u } / \mathrm{w}^{1 /} / \text { particule d'interrogation }
\end{aligned}
$$

Les phonèmes brefs $/ \gamma /$ et $/ a ̆$ sont issus de leur correspondants $/ \gamma /$ et $/ \mathrm{a} /$.

Les autres phonèmes- voyelles d'aperture non-fermée pourraient être brefs et que leur manifestation n'est qu'occasionnelle, lors de leur combinaison avec d'autres phonèmes. Alors les durées de $/ \mathrm{o} /$, de $/ \mathrm{o} /$, de /e/, de / $\varepsilon$ / sont aussi brèves les unes que les autres; on ne peut constater clairement que lorsqu'il existe des paires minimales comme dans toong/ tong, lanh canh/ leng keng...

A côté des voyelles dites simples, il existe en vietnamien trois diphtongues /ie/, /u//, /uo/ (l'existence de ces trois diphtongues est discutée car les uns les considèrent comme diphtongues, les autres comme groupes de phonèmes distincts). Quand ils ne sont pas suivis par une finale, ils s'approchent phonétiquement et phonologiquement de /a/- autre phonème central plus ouvert qu'eux.

$$
\begin{gathered}
\text { Exemple: tiếng/ tía } \\
\text { lườn/ lừa } \\
\text { buôn/ búa... }
\end{gathered}
$$

\section{Etudes contrastives des systèmes vocaliques français et vietnamien}

Les systèmes vocaliques français et vietnamien comportent à la fois des phonèmes communs et différents. En comparaison de ces deux systèmes, on peut tirer quelques remarques: 


\subsection{Points communs}

Le français et le vietnamien utilisent en commun 7 voyelles: /i/, /e/, / $/$ /, /a/, /o /, /o/, /u/ (on distingue en français comme en vietnamien, par des critères de lieux d'articulation et d'aperture, des voyelles plus ou moins antérieures ou postérieures et des voyelles plus ou moins fermées ou ouvertes).

Ex: [de]: en vietnamien $\rightarrow$ digue (đê)

en français $\rightarrow$ article défini pluriel (des), petit cube dont chaque face est marquée d'un nombre différent de points, de un à six, et servant à des jeux de hasard (dé)

On se rend compte qu'en vietnamien, il n'y a qu'une façon d'écrire un son, tandis qu'en français, plusieurs graphies peuvent correspondre à un seul son.

\subsection{Points distinctifs}

Le système vocalique vietnamien possède quelques traits distinctifs que ne connaît pas le système vocalique français: la stabilité du timbre et la durée des voyelles:

On parle vietnamien avec six différents accents tandis qu'en français, il n'y a qu'un seul. Grâce à la variété des accents, les tons en vietnamien connaissent plus de stabilité.

En français, la durée n'est pas un trait phonologique. En vietnamien, l'opposition entre "voyelle brève" et "voyelle longue" garde toute sa valeur. On distingue par exemple les voyelles brèves $/ a \mathrm{a} /, / \gamma /$ qui s'opposent respectivement à leurs homologues longues $/ \mathrm{a} /, / \gamma /$. Les voyelles brèves en vietnamien ne peuvent se réaliser que quand elles sont accompagnées d'une consonne finale. On peut citer également des oppositions: $/ 0 \%-/ 0 /$ (xong- xoong), $/ \varepsilon \%-/ \varepsilon /$ (sành- xèng)

Puis, en vietnamien, le noyau syllabique d'un mot peut comporter plus d'une voyelle qui se groupent en une seule émission de voix: on est en présence d'une diphtongue:

$/ \mathrm{u} /+/ \mathrm{o} / \rightarrow / \mathrm{uo} / \ldots$

A la différence du vietnamien, langue tonale, le français a, lui aussi, des traits particuliers. Pour aider les apprenants vietnamiens du français, il nous suffit de nous intéresser à des points particuliers du français:

\subsubsection{Voyelles arrondies}

Le français exploite à un degré poussé la labialisation. Les voyelles arrondies sont:

- les postérieures arrondies (comme en vietnamien): /u/,/o/,/0/

$$
\begin{aligned}
\text { Ex: } / \mathrm{u} /: \operatorname{doux}[\mathrm{du}] \\
/ \mathrm{o} / \mathrm{:} \text { écho [eko] } \\
\text { /o /: Nord [no R] }
\end{aligned}
$$

- les antérieures arrondies (qu'on ne trouve pas en vietnamien): /y/, /f/, /œ/

$$
\begin{gathered}
\text { Ex: /y/: numéro [nymeRo] } \\
\text { durée [dyRe] } \\
\text { /ø/: bleu [blø] } \\
\text { peu [pø] } \\
\text { /œ/: } \text { cœu r [kœR] } \\
\text { heure [œR] }
\end{gathered}
$$

- la voyelle médiane arrondie: /ə / et la nasale arrondie / \% \% 


$$
\begin{array}{r}
\text { Ex: /ə /: le degré [lə də gRe] } \\
\text { le renard [lə Rə naR] }
\end{array}
$$

Parmi elles, les trois voyelles antérieures arrondies sont en constante opposition avec trois voyelles antérieures écartées: /y/, /ø/, /œ/ s'opposent à /i/,/e/, /ع/.

\subsubsection{Voyelles nasales}

Le français utilise la nasalisation qui n'existe pas dans le système vocalique vietnamien. En français, il y a quatre voyelles nasales:

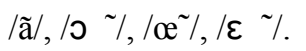

$$
\begin{aligned}
& \text { Ex: /ã/: vacances [vakãs] } \\
& \text { temps [tã] } \\
& \text { /० /: savon [savo ] } \\
& \text { monde [mo d] } \\
& / œ /: \text { parfum [paRfœ ] } \\
& \text { aucun [okœ ] } \\
& / \varepsilon \% \text { : pain [pe ] } \\
& \text { sympathie [sع pati] }
\end{aligned}
$$

On confond souvent les voyelles nasales en français avec le groupe "voyelles brèves + consonne finale nasale $/ \eta /$ palatalisée" en vietnamien. Les voyelles nasales en français $/ \tilde{a} /, / 0 \%$ et $/ \varepsilon \%$ se réaliseront respectivement comme /ăn/ (ăng), /on/ (ông) et /en/ (anh) en vietnamien. La voyelle nasale / /e / en français est souvent identifiée par les Vietnamiens au groupe $/ 0 \eta$ / (oong) de leur langue. Le problème de la nasalisation pose, comme on verra plus loin, d'énormes difficultés pour les apprenants vietnamiens.

\subsubsection{Semi-voyelles ou semi-consonnes}

Nous pouvons voir de ce qui a été dit que la distinction entre voyelles et consonnes n'est pas absolue. Une voyelle peut être prononcée seule en français alors qu'une consonne, en général, doit être accompagnée d'une voyelle pour être prononcée. Cela n'est vrai que pour les occlusives et sonantes. Mais les fricatives n'ont pas besoin de s'appuyer sur une voyelle. On peut même "chanter" avec le /z/. On peut dire que ces consonnes ont suffisamment de son en elles-mêmes. D'autre part, quand nous prononçons $/ \mathrm{i} /, / \mathrm{y} /, / \mathrm{u} /, \mathrm{des}$ voyelles qui sont très fermées, nous voyons qu'il suffit de relever un petit peu la langue et réduire ainsi leur aperture pour obtenir des semi-voyelles ou semi-consonnes.

$$
\begin{gathered}
\text { Ex: /j/: pied [pje] } \\
\text { viande [vjãd] } \\
\text { /w/: loin [lwẽ] } \\
\text { moi [mwa] } \\
/ \mathrm{Y} /: \text { sueur [su œR] } \\
\text { enfuir [ãfu iR] }
\end{gathered}
$$

\subsubsection{Attaque vocalique}

A part ces différences observées sur le plan articulatoire (phonologique), on remarque d'autres divergences concernant par exemple l'attaque vocalique et le mode de combinaison. 
En vietnamien, l'attaque vocalique est beaucoup plus forte qu'en français. Les frontières syllabiques étant "solides", toute influence combinatoire est impossible en cette langue. Ainsi, le vietnamien ne connaît pas l'enchaînement vocalique: une syllabe attend toujours l'extinction complète du dernier phonème de la syllabe précédente pour commencer. Toute syllabe vietnamienne commençant par une voyelle est toujours attaquée par un coup de glotte pareil à une articulation consonantique.

$$
\text { Exemple: [? } \left.\mathrm{m}^{5} 7 a \mathrm{ă}^{1} 7 \mathrm{ok}^{5} 7 a \mathrm{n}^{1} 7 \mathrm{k}^{5}\right] \text { (ứ ăn ốc ăn ếch) }
$$

En français, la situation est tout à fait différente. L'enchaînement vocalique et consonantique est une règle fondamentale à respecter pour assurer le débit normal de la chaîne parlée. Les voyelles et consonnes se suivent automatiquement dans l'énonciation sans devoir attendre la chute totale de l'une pour commencer l'autre. Cette habitude articulatoire constitue un grand obstacle pour l'apprenant vietnamien.

\section{Correction phonétique}

Lors de l'apprentissage de langues étrangères, la prononciation correcte est nécessaire. L'enseignement de la prononciation devrait impliquer la correction phonétique. Au vietnam, on néglige encore, malheuresement, des problèmes de l'enseignement phonétique en général et de la correction phonétique en particulier.

Parallèllement aux méthodes traditionnelles de correction phonétique, telles que méthode articulatoire, méthode basée sur l'audition consciente, méthode verbo-tonale, nous souhaitons exposer ici nos propres méthodes de correction phonétique, conçues exclusivement pour les apprenants vietnamiens du français langue étrangère: A partir de l'analyse contrastive des systèmes vocaliques du français et du vietnamien, nous allons dégarer grosso modo les difficultés les plus importantes contre lesquelles les apprenants vietnamiens du français peuvent éventuellement se heurter. Dans le cadre restreint de cet article, nous nous contentons de traiter à priori les fautes des voyelles que l'apprenant vietnamien commet souvent.

\subsection{Voyelles en commun des deux langues}

On a 7 voyelles que les deux systèmes utilisent en commun: /i/, /e/, / $\varepsilon /$, /a/, /o /, /o/, /u/. Pourtant, la graphie de chaque langue n'est pas toujours la même, l'apprenant vietnamien a toujours tendance à articuler à la vietnamienne le français.

Ex: Avec la graphie "gai", on prononce en français /ge/ (cela signifie "amusant", adjectif masculin), mais tout en regardant cette graphie, certains apprenants vietnamiens prononcent/gaj/, ce mot veut dire "épine" en vietnamien.

\subsection{Voyelles propres du français}

\subsubsection{Voyelles antérieures arrondies}

Les trois voyelles antérieures arrondies sont en constante opposition avec trois voyelles antérieures écartées: /y/, /Ø/, /œ/ s'opposent à /i/, /e/, /E /. En vietnamien, comme cette opposition de labialisation n'existe pas, quand il perçoit et produit ces voyelles antérieures arrondies, l'apprenant vietnamien a tendance à leur attribuer, à tort certes, des caractéristiques des autres voyelles de son système linguistique.

Au lieu de prononcer /y/, /ø/, /œ/, ils prononcent /u/ (ou "uy"), / $/ /$ :

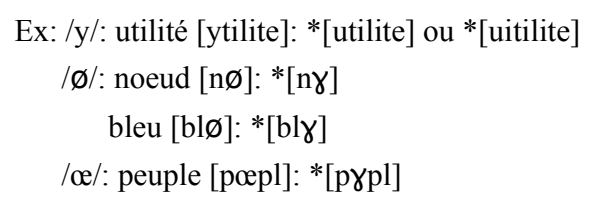


oeuf [œf]: *[rf]

Pour la voyelle /y/, il y a deux tendances:

La faute peut venir de la méconnaissance de la graphie utilisée. En vietnamien, la lettre "u" doit se prononcer [u]. En voyant "pu" par exemple, il le prononce automatiquement comme "tout". Cependant, cette faute est due à l'orthographie.

$$
\begin{aligned}
& / \mathrm{y} /: \text { rue }[\mathrm{Ry}]: *[\mathrm{Ru}] \\
& \text { numéro [nymeRo]: } *[\text { numeRo] }
\end{aligned}
$$

La deuxième tendance est que l'apprenant identifie souvent /y/ au groupe /uj/ qui existe en vietnamien "uy". C'est la faute la plus fréquente. C'est justement le caractère intermédiaire de /y/ (situé entre /i/ et /u/ qui provoque la faute).

$$
\begin{aligned}
& / \mathrm{y} /: \text { rue }[\mathrm{Ry}]: *[\mathrm{Rui}](\text { en vietnamien) } \\
& \text { numéro [nymeRo]: * [nuimeRo] (en vietnamien) }
\end{aligned}
$$

En français, il y a trois phonèmes $/ \varnothing /$, /ə /, /œ/ qui sont des phonèmes non fermés (par rapport à /i/, /y/, $/ \mathrm{u} /$ ), non ouverts (par rapport à /a/, /ã), pas très antérieurs (par rapport à /i/, /e/, / $/$ /) et non postérieurs (par rapport à $/ \mathrm{u} /, / \mathrm{o} /, / \mathrm{o} /$ ). En vietnamien, le seul phonème qui présente les mêmes similitudes est le phonème $/ \gamma /$. Cependant, alors que:

$+/ \varnothing /$ et /œ/ tendent vers l'avant, $/ \gamma /$ tend vers l'arrière.

$+/ \varnothing /$ et /œ/ sont des arrondis, $/ \gamma /$ est non arrondi.

D'ailleurs, certains apprenants vietnamiens, étant moins sensibles à la différence d'aperture entre $/ \varnothing / \mathrm{mi}$ fermé, /ə / moyen et / a / mi-ouvert, les réalisent comme un seul, proche du phonème $/ \gamma /$ de leur langue maternelle.

Il nous semble absolument nécessaire de corriger les fautes qui concernent les deux phonèmes /ø/ et /œ/. Les fautes de /ə / (confusion avec $/ \gamma /$ ) ne fausseront pas la communication, ne sont pas bien analysées.

\subsubsection{Voyelles nasales}

En vietnamien, il y a seulement les voyelles buccales, tandis qu'en français, il y en a quatre dites nasales: $/ \tilde{a} /, / 0 \%, / \mathfrak{e}^{2} /, / \varepsilon \%$. Cela cause aux apprenants d'énormes difficultés en articulant les voyelles nasales: L'apprenant vietnamien a tendance à assimiler les voyelles nasales françaises (/ã/, /0 \%, / $\mathfrak{e}^{\sim} /, / \mathcal{\varepsilon} /$ ) aux sons buccaux vietnamiens (souvent "une voyelle brève" + "la consonne nasale / $\mathfrak{n}$ palatalisée").

On peut trouver des fautes portant sur les voyelles nasales dans les corpus suivants:

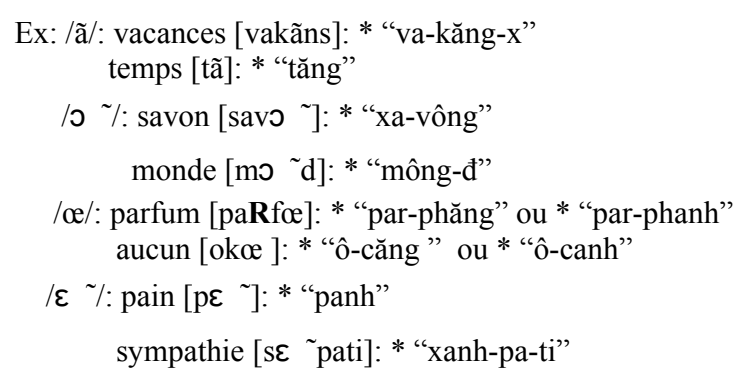




\subsubsection{Semi-voyelles}

L'apprenant vietnamien commet également des fautes concernant les semi-voyelles /j/, / $/$ /:

-Semi-voyelle /j/:

/j/ à l'initiale et au milieu de la syllabe:

$$
\begin{aligned}
& \text { hier [jeR]: * [ieR] } \\
& \text { yeux [jf]: * [if] } \\
& \text { viande [vjãd]: * [viãd] } \\
& \text { billet [bije]: * [biie] }
\end{aligned}
$$

Le semi-voyelle /j/ n'est jamais à l'initiale ni à l'intérieur de la syllabe en vietnamien. L'apprenant vietnamien ne sait pas donc comment la prononcer dans ce cas ou bien il prononce comme une voyelle et non comme une semi-voyelle /j/ à la fin de la syllabe:

Quand elle est à la finale, il préfère ou bien la laisser tomber complètement, ou bien la réaliser sans la phase d'explosion finale, ou encore ajouter une voyelle d'appui qui est la voyelle $/ \gamma /$ pour former une nouvelle syllabe:

$$
\begin{aligned}
& \text { fille [fij]: * [fi] ou * [fir] } \\
& \text { nouille [nuj]: * [nui] ou * [nuið] }
\end{aligned}
$$

-Semi-voyelle / / /:

L'apprenant vietnamien du français commet souvent une faute commune:

$$
\begin{aligned}
& \text { sueur [su œR]: * [suiøR] } \\
& \text { lui [ly i]: * [lui] }
\end{aligned}
$$

\subsubsection{Attaque vocalique}

Lorsqu'un mot se termine par une voyelle prononcée et que le mot suivant commence par une voyelle, en français, il n'y a pas d'arrêt de la voix entre les deux voyelles: les deux voyelles sont enchaînées.

\begin{tabular}{|c|c|c|}
\hline & en français & en vietnamien \\
\hline$/ \mathrm{a} /$ & a, â, ea, oi, e $(+\mathrm{mm})$, oy, oe, $\ldots$ & $\mathrm{a}$ \\
\hline \multicolumn{3}{|c|}{ Ex: fade, pâle, mangea, moi, femme, moyen, moelle, ... } \\
\hline /e/ & é, ée, et, ez, es, eai, aie, ais, ait, ae,... & $\hat{\mathrm{e}}$ \\
\hline \multicolumn{3}{|c|}{ Ex: salé, poupée, et, avez, des, mangeait, CV... } \\
\hline /e/ & e, ở, ê, è, ès, ai, aợ .... & $\mathrm{e}$ \\
\hline
\end{tabular}
Pourtant, l'apprenant vietnamien du français ajoute toujours un coup de glotte avant chaque voyelle initiale:

$$
\text { J'ai eu un billet [3eyœbije]: * [?ze?y?œ?bi?je] }
$$

\section{Propositions méthodologiques}

\subsection{Fautes des voyelles en commun des deux langues}

Puisqu'une même graphie peut être prononcée de façons très différentes dans le français et dans le vietnamien. Il faut, tout d'abord, indiquer à l'apprenant vietnamien la différence entre la graphie et le son. 


\begin{tabular}{|c|c|c|}
\hline \multicolumn{3}{|c|}{ Ex: bec, Noël, blême, poète, dès, aime, fraïche, ... } \\
\hline /i/ & $\mathrm{i}, \hat{\mathrm{i}}, \mathrm{ù}, \mathrm{y}, \ldots$ & $\mathrm{i}, \mathrm{y}$ \\
\hline \multicolumn{3}{|c|}{ Ex: ski, île, Hanoù, stylo, ... } \\
\hline /0 / & $\mathrm{o}, \mathrm{au}$ & $\mathrm{o}$ \\
\hline \multicolumn{3}{|c|}{ Ex: note, robe, mode, col, Paul, ... } \\
\hline$/ \mathrm{o} /$ & $\mathrm{o}, \hat{o}, \mathrm{au}, \mathrm{aux}, \mathrm{eau} . .$. & $\hat{o}$ \\
\hline \multicolumn{3}{|c|}{ Ex: sot, drôle, aube, taux, agneau, ... } \\
\hline$/ \mathrm{u} /$ & ou, où, -oup, -oût, aoû, ... & $\mathrm{u}$ \\
\hline
\end{tabular}

La correction des voyelles en commun en deux langues ne reste que dans la pure attention de la graphie et du son des mots prononcés.

\subsection{Fautes des voyelles antérieures arrondies}

Pour corriger les fautes des voyelles antérieures arrondies, l'enseignant doit montrer à l'apprenant la différence des graphies et des sons entre les deux langues. Ensuite, on suit cette règle générale:

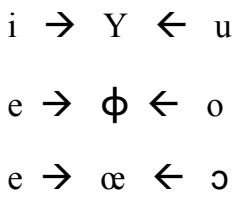

-Pour la voyelle /y/: D'abord, l'enseignant demande à l'apprenant de prononcer /i/, puis de maintenir la position de la langue en arrondissant graduellement les lèvres comme dans la prononciation de $/ \mathrm{u} /$. La différence fondamentale entre [ly] et [luj] réside dans ce que quand on prononce [ly], les lèvres restent arrondies depuis le début jusqu'à la fin de l'émission, tandis que quand on prononce [luj] ("luy" en vietnamien), les lèvres deviennent écartées vers la fin de l'émission. Pour obtenir le [y] français, les lèvres doivent rester arrondies depuis le commencement jusqu'à la fin de l'émission de la voyelle.

D'autre méthode pour la voyelle $/ \mathrm{y} /:[\mathrm{y}]$ et $[\mathrm{u}]$ sont tous les voyelles arrondies, les lèvres sont avancées dans la même position. Mais elles s'opposent ainsi: [y] est une voyelle antérieure, [u] est une voyelle postérieure qui existe dans le vietnamien. Donc, nous commençons à articuler [u], puis laissons avancer la langue.

-Pour les voyelles $/ \phi /$ et /œ /: Si l'apprenant vietnamien réalise $[\phi]$ et [œ] comme $[\gamma]$ vietnamien, c'est que du point de vue articulatoire, d'une part sa langue est trop reculée en arrière, d'autre part ses lèvres ne sont pas assez arrondies. Pour corriger les fautes de ce genre, nous pouvons appliquer une des méthodes suivantes:

+Commencer par articuler [o] et [0 ]:

Pour le son [\$]: L'enseignant montre à l'apprenant vietnamien l'opposition entre [o] (qui existe en français et en vietnamien) et [\$] (qui n'existe qu'en français). [o] et [\$] ont des points communs: Quand nous les articulons, les mâchoires sont presque fermées, les lèvres sont arrondies. La position de la langue pendant l'articulation est la seule différence entre ces deux sons: La langue est en arrière dans le cas de

[o] et en avant dans le cas de [\$]. Alors, l'apprenant articule d'abord le son [o], puis il maintient la 
position des lèvres en poussant la langue en avant (comme la position de la langue de [e] ), l'apex de la langue touchant les dents inférieurs.

Pour le son [œ]: L'apprenant peut appliquer la même règle générale avec [0 ], puis pousse la langue en avant (comme la position de la langue de $[\varepsilon]$ )

+ Commencer par articuler $[\mathrm{e}]$ et $[\varepsilon]$ :

Pour le son $[\phi]$ : L'apprenant prononce [e], maintient la position de la langue tout en arrondissant les lèvres (comme la position des lèvres de la voyelle $[\mathrm{o}]$ )

Pour le son [œ]: L'apprenant prononce [e], maintient la position de la langue tout en arrondissant les lèvres (comme la position des lèvres de la voyelle [0 ])

\subsection{Fautes des voyelles nasales}

Les voyelles nasales françaises n'existent pas en vietnamien. Alors, il faut, tout d'abord, indiquer à l'apprenant vietnamien la différence entre la graphie et le son de chaque voyelle nasale française:

\begin{tabular}{|c|c|}
\hline$/ \tilde{a} /$ & am, an, em, en, ean, aon, ang \\
\hline \multicolumn{2}{|r|}{ Ex: champs, tante, temps, sentir, Jean, paon, étang... } \\
\hline / $\%$ & on, om \\
\hline \multicolumn{2}{|r|}{ Ex: bonjour, maison, concombre, tomber, ... } \\
\hline$/ \mathfrak{e}^{\sim} /$ & un, um, eun \\
\hline \multicolumn{2}{|r|}{ Ex: aucun, parfum, à jeûn, ... } \\
\hline$/ \varepsilon \sim$ & in, im, ain, aim, ein, ym, en \\
\hline & Ex: dinde, timbre, main, faim, freins, sympathie, bien, ... \\
\hline
\end{tabular}

Et puis pour l'entraînement de ces voyelles, l'enseignant doit montrer à l'apprenant la différence entre une voyelle nasale française et sa fausse articulation correspondante en vietnamien: L'apprenant vietnamien remplace souvent la voyelle nasale française par une voyelle buccale vietnamienne et la consonne nasale vietnamienne $/ \eta /$. La faute est assez grave parce qu'une voyelle et une consonne sont très différentes:

+Une voyelle est produite par le courant d'air qui passe librement à travers les résonateurs sans être arrêté par aucun organe.

+ Pour produire une consonne, certains organes doivent se joindre pour faire obstacle au passage de l'air (il s'agit d'un temps d'implosion et d'un temps d'explosion).

Alors, tandis que la prononciation juste finit par une voyelle et aucun organe buccal n'est bloqué; en réalité, elle se termine mal par la consonne $/ \mathfrak{\eta} /$, vélaire et nasale.

$$
\begin{aligned}
& \text { Ex: /ã/: vacant [vakã]: * [vakăh }{ }^{11} \text { ] } \\
& 10 \text { \%: savon [savo ]: * [xa vôn] }
\end{aligned}
$$

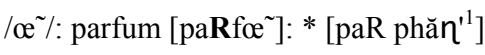

$$
\begin{aligned}
& / \varepsilon \% \text { : pain [pe }]: *^{*}\left[p^{1} \eta^{1}\right]
\end{aligned}
$$


Pour corriger cette faute, il faut d'abord enlever la consonne nasale / $\mathfrak{n}$. Pour cela, l'enseignant demande à l'apprenant de tenir la voyelle pendant son émission sans changer la position de la langue et des lèvres, puis de la terminer nettement, sans ajouter la consonne nasale $/ \eta /$ :

$$
\begin{aligned}
& \text { * "vông" [von'1] } \rightarrow \text { "vôôô ô ô ..." [vo] }
\end{aligned}
$$

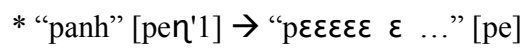

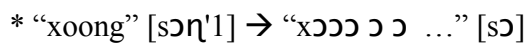

Ensuite, l'enseignant demande à l'apprenant de nasaliser la voyelle orale prononcée tout en laissant passer une partie du courant d'air qui vient des poumons par les fosses nasales. Ceci n'est pas évident. Pour faciliter le passage par le nez, il faut:

+soit entraîner avec les consonnes nasales $/ \mathrm{m} /, / \mathrm{n} /, / \mathrm{n} /$ :

$$
\begin{aligned}
& \text { "vôôô ô ô ..." [vo] } \rightarrow \text { "môôô ô ô ..." [mo] } \rightarrow \text { [mo ] }
\end{aligned}
$$





+soit se référer à l'audition: écouter l'enregistrement des natifs.

\subsection{Fautes des semi-voyelles}

On peut représenter les principales caractéristiques articulatoires dans le tableau suivant:

\begin{tabular}{|l|l|l|}
\hline Antérieure écartée & antérieure arrondie & postérieure arrondie \\
\hline$/ \mathrm{j} /$ & $/ \mathrm{U} /$ & $/ \mathrm{w} /$ \\
$\begin{array}{l}\text { langue avancée } \\
\text { lèvres écartées }\end{array}$ & $\begin{array}{l}\text { langue avancé } \\
\text { lèvres arrondies }\end{array}$ & $\begin{array}{l}\text { langue reculée } \\
\text { lèvres arrondies }\end{array}$ \\
\hline
\end{tabular}

Le yod $/ \mathrm{j} /$, articulé comme $/ \mathrm{i} /$, peut se trouver avant ou après une voyelle

$$
\text { Ex: /j/: travail [tRavaj] }
$$

Notons la différence entre "paye" [pej] (une seule syllabe) et "pays" [pei] (2 syllabes)

Le son noté /w/ est articulé comme /u/ mais lié étroitement à la voyelle suivante/

$$
\text { Ex: /w/: loin [lwe] }
$$

Enfin, le son noté /y / s'articule comme /y/ et est étroitement liée à la voyelle qui suit.

$$
\text { Ex: } \quad \text { enfuir [ãfu } i R]
$$

Attention: quand on a une combinaison:

$$
\text { Consonne + "L" ou "R" + "ou" + voyelle }
$$

on a deux syllabes:

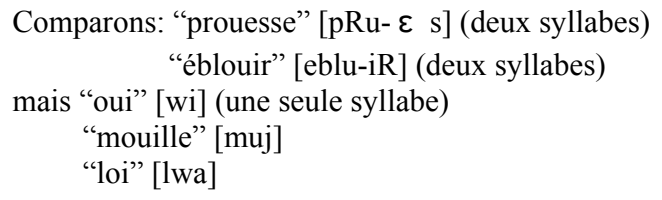


Pourtant, pour faciliter encore le processus de correction, l'apprenant vietnamien peut suivre les conseils suivants:

\subsubsection{Semi-voyelle /j/}

+Quand elle n'est pas à la finale, l'apprenant vietnamien a tendance à la prononcer comme une voyelle et non comme une semi-voyelle. Ce n'est pas une faute très grave car la yod $/ \mathrm{j} /$ se prononce à peu près comme le “i”, mais il est toujours suivi ou précédé d'une voyelle, et

-il s'articule avec la langue plus fortement appuyée contre le palais que pour le " $\mathrm{i}$ ".

-il s'articule plus longuement.

$*$ [siel $] \rightarrow[$ sjel] "cieeel"

* [biie] $\rightarrow$ [bije] "biiiillet"

+Quand elle est à la finale, l'apprenant commet souvent des fautes suivantes:

$$
\begin{aligned}
& \text { fille [fij]: * [fi] ou } *[\text { fij }] \\
& \text { nouille [nuj]: * [nui] ou } * \text { [nuj ] }
\end{aligned}
$$

S'il est réalisé sans la phase d'explosion, il faudra chercher à ouvrir un peu la syllabe. En effet, on pourra, par exemple, abaisser la mâchoire inférieure, laissant ainsi s'échapper un peu d'air après la phase d'implosion:

$$
\begin{aligned}
& {[\mathrm{fi}] \rightarrow[\mathrm{fij}]} \\
& {[\text { nui }] \rightarrow[\text { nuj }]}
\end{aligned}
$$

Si l'apprenant ajoute à tort une voyelle d'appui $/ \gamma /$, il est évident qu'on doit chercher à terminer la fin de la syllabe par une fermeture. Alors, nous passons de la voyelle ouverte à celle fermée et l'enlevons:

$$
\begin{array}{ll}
\text { Fautes: } & \multicolumn{1}{c}{\text { Procédés proposés: }} \\
{[\text { fij }]} & -->[\text { fije] }->\text { [fiji] --> [fij] } \\
{[\text { nuj }]} & -->[\text { nuje] }-->[\text { nuji }]-->\text { [nuj] }
\end{array}
$$

\subsubsection{Semi-voyelle /4 I}

Pour la semi-voyelle / $/$ /, il s'agit d'un nouvel enseignement. Nous pensons que si l'apprenant arrive à bien prononcer le phonème $/ y /$, il parviendra sans beaucoup de difficultés à prononcer $/ 4$ / comme il faut. Les procédés de correction appliqués pour $/ 4$ / sont à peu près les mêmes que ceux appliqués pour $/ \mathrm{y} /$. Pour les Vietnamiens, ce qui s'avère le plus important, c'est de savoir tenir les lèvres arrondies pendant toute la phase d'émission.

Pour corriger cette faute, d'abord l'apprenant devra séparer temporairement les deux voyelles voisines:

$$
\begin{aligned}
& \text { tuer [ty e] } \rightarrow[\text { ty }]+[\text { e }] \\
& \text { lui }[\text { ly i] } \rightarrow[\text { ly }]+[i]
\end{aligned}
$$

Ensuite, il prononce la première voyelle ([ty] ou [ly]) tout en faisant maintenir que les lèvres ne deviennent pas écartées.

Enfin, il glisse la prononciation de la première voyelle à la seconde ([e] ou [i]). L'importance consiste à relier les prononciations de ces deux voyelles dans un seul souffle, sans aucune rupture au niveau acoustique. 


\subsubsection{Semi-voyelle $/ \mathrm{w} /$}

Pour la semi-voyelle /w/, la situation est plus simple. Du point de vue articulatoire, il n'y a pas d'interférences particulières. Le phonème possède presque les mêmes qualités dans une langue que dans l'autre. Il nous faut seulement remarquer la présence de la diphtongue /uo/ en vietnamien. Ce phonème posera plus de difficultés pour les Français apprenant le vietnamien que pour les Vietnamiens apprenant le français.

Pour mieux prononcer cette semi-voyelle, il nous suffit de tenir la voyelle plus longtemps parce que pour les Indochinois, la voyelle qui suit le /w/ est en général trop courte.

En tenant compte de toutes ces fautes, l'apprenant peut s'entraîner avec le miroir.

\subsection{Fautes de l'attaque vocalique}

L'attaque vocalique est souvent très dure en vietnamien. Pour adoucir une pareille attaque de la voyelle initiale en franỗais, l'enseignant demande à l'apprenant de commencer par une légère aspiration avant d'attaquer la voyelle en question:

$$
\begin{aligned}
& \text { Ex: }- \text {------> elle est belle } \\
& \quad----->\text { allons-y, nos enfants? } \\
& \quad----->\text { et avec qui alors? }
\end{aligned}
$$

Un autre procédé préconise l'allongement de la voyelle initiale pendant un certain temps:

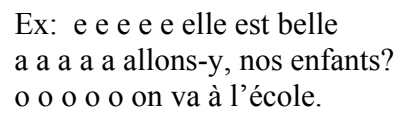

Une dernière proposition consiste à placer des mots commençant par une voyelle dans les contextes où il y a liaison et enchaînement:

$$
\begin{aligned}
& \text { Ex: Vas-y! } \\
& \text { Quand elle ira. } \\
& \text { Il a neuf ans. }
\end{aligned}
$$

Nous pensons que dans ces cas présents, une explication de la part de l'enseignant est utile et qu'elle aide ce dernier à gagner du temps.

\section{Conclusion}

"Comparaison n'est pas raison", aucune comparaison n'est parfaite. De plus, le français est une langue flexible tandis que le vietnamien est isolant. La problématique reste bien peu réaliste pour les Vietnamiens.

A travers la recherche, nous cherchons à profiter de caractères communs des deux systèmes vocaliques français et vietnamien, à distinguer clairement les particularités en vue de les aider à bien articuler les sons du français.

Nous avons examiné de près les fautes des voyelles qui sont éventuellement nées des interférences entre le français et le vietnamien, abordé à priori quatre grandes difficultés concernant les voyelles nasales, les voyelles antérieures, les semi-voyelles et l'enchaînement vocalique et proposé nos propre méthodes de correction pour l'apprenant vietnamien.

Un vrai débutant commet en même temps de nombreuses fautes. L'intervention immédiate du professeur est nécessaire mais il ne devrait pas consacrer tout son temps à la correction phonétique, c'est à dire que nous, les professeurs de FLE, pouvons choisir, pour un certain temps, les fautes les plus graves à corriger. Pour la correction phonétique, nous devons être patients. L'impatience n'apporte pas certainement de bons résultats. 


\section{Ouvrages de référence}

Charliac, L., Morton, A-C. (1998). Phonétique progressive du français- Avec 600 exercices, CLE International.

Cu Đình Tú (1990). Ngũ âm tiếng Việt hiện đại, Hà Nội : Maison d'édition de l'Education.

Doan Thiện Thuật (1977). Ngũ âm tiếng Việt, Hà Nội : Maison d'édition de l'Université et du Lycée professionnels.

Léon, M. (1964). Exercices systématiques de prononciation française, Paris : Librairies Hachette et Larousse,.

Lévi-Strauss, C. (1938). Anthropologie structurale, Paris.

Nguyen Thiện Giáp (1998). Linguistique, Hà Nội : Maison d'édition de l'Education.

Nguyen Thị Thu Hường (2006). Linguistique appliquée, Hà Nội : ELSE- UNH.

Nguyen Hữu Quỳnh (1979). Cơ sở ngôn ngũ họ II, Hà Nội : Maison d'édition de l'Education.

Nguyen Lân Trung (1984-1985). De la linguistique contrastive à l'élaboration d'un manuel de français langue étrangère- Une application à la réalité vietnamienne, Hà Nội : ESLE-UNH.

Pottier, B. et des auteurs (1973). Le langage- Encyclopédie du savoir moderne, Paris.

Wioland, F. (1991). Prononcer les mots du français, Hachette F.L.E. 